\title{
Handlungsfelder und Arbeitsbereiche nach dem dualen Pflegestudium - Eine Verbleibstudie an der FH Münster
}

\author{
Ailina Christina Claaßen ${ }^{1}$ Katharina Jeiler ${ }^{1}$ Dorothee Martens ${ }^{1} \cdot$ Claudia Oetting-Roß $^{1}$
}

Eingegangen: 21. Juli 2020 / Angenommen: 19. Februar 2021 / Online publiziert: 18. März 2021

(c) Der/die Autor(en) 2021

\section{Zusammenfassung}

Hintergrund Nach der Etablierung grundständiger Studiengänge in der Pflege stehen die ersten Absolvent_innen inzwischen dem Arbeitsmarkt zur Verfügung. Unabhängig vom erwarteten Mehrwert für die Pflegepraxis sind einige Aspekte hinsichtlich des beruflichen Verbleibs, der Aufgabenprofile und Handlungsfelder sowie der beruflichen Zufriedenheit nach wie vor unklar.

Ziel der Arbeit Die vorliegende Arbeit beschäftigt sich mit der Frage, in welchen Handlungsfeldern und Arbeitsbereichen Absolvent_innen eingesetzt werden, wie zufrieden sie damit sind, und welche akademischen Weiterqualifizierungen sie in Anspruch nehmen bzw. anstreben.

Methodik Nach einer literaturbasierten Fragebogenentwicklung erfolgte die Datenerhebung als onlinegestützte Befragung (EvaSys) der bisherigen Absolvent_innenjahrgänge des Bachelor-Studiengangs Pflege dual der FH Münster. Die Befragungsergebnisse wurden deskriptiv mittels SPSS ausgewertet.

Ergebnisse Die Ergebnisse zeigen ein vielfältiges Aufgabenprofil in der direkten pflegerischen Versorgung mit überwiegend klientennahen Tätigkeiten. Die Befragten sind insgesamt eher zufrieden mit ihrer beruflichen Tätigkeit. Zudem verdeutlicht die Aufnahme verschiedener aufbauender Studiengänge ein Streben nach Weiterqualifizierung.

Schlussfolgerung Den Absolvent_innen gelingt es in der Regel gut, sich Handlungsfelder und (erweiterte) Arbeitsbereiche im Rahmen ihrer Pflegetätigkeit zu erschließen. Es scheint, als erfolge diese Schaffung neuer Tätigkeitsfelder nicht selten durch die Eigeninitiative der Absolvent_innen selbst. Dies impliziert die Notwendigkeit einer Weiterentwicklung der beruflichen Praxis, insbesondere hinsichtlich spezifischer Stellenprofile und Personalkonzepte für akademisch qualifizierte Pflegefachkräfte. Die Entwicklung passgenauer Stellenprofile sollte die berufliche Verortung der Absolvent_innen zukünftig unterstützen und ihnen schon im Studium eine Perspektive bieten.

Schlüsselwörter Berufsverbleib $\cdot$ Akademisierung der Pflege $\cdot$ Pflege $\cdot$ Duales Pflege-Studium $\cdot$ Karriere

\section{Fields of activity and work areas after dual nursing studies-A retention study at Münster University of Applied Sciences}

\begin{abstract}
Background Following the establishment of undergraduate degree courses in nursing, the first graduates are now available on the labor market. Irrespective of the expected added value for nursing practice, some aspects remain unclear with respect to career retention, task profiles and fields of activity as well as professional satisfaction.

Aim The present study deals with the question, in which fields of work graduates are employed, how satisfied they are with them and which further academic qualifications they take up or strive for.

Method After a literature-based questionnaire development, the data collection was carried out as an online-supported survey of the previous graduates of the B. Sc nursing dual course at the FH Münster University of Applied Sciences. The survey results were descriptively analyzed using SPSS.
\end{abstract}

Ailina Christina Claaßen

ailina.claassen@fh-muenster.de
1 Fachbereich Gesundheit (MSH) - FH Münster, Leonardo Campus 8, 48149 Münster, Deutschland 
Results The results show a diverse job profile in direct nursing care with predominantly client-related activities. Respondents were relatively satisfied with their professional activities. In addition, the inclusion of several postgraduate courses indicates a desire for further qualifications.

Conclusion Among others, graduates generally succeeded well in developing fields of activity and (extended) work areas in the context of nursing profession. It seems that the creation of new fields of activity is often the result of the graduates' own initiative. This implies the need for further development of professional practice, especially with respect to specific job profiles and personnel concepts for academically qualified nurses. The development of job profiles tailored to the needs of the graduates should support their professional positioning in the future and offer them a perspective even during their studies.

Keywords Occupational retention $\cdot$ Nursing academization $\cdot$ Nursing $\cdot$ Integrated degree program $\cdot$ Career

\section{Hintergrund}

Die Professionalisierung der Pflegefachkräfte hat in den letzten Jahren durch den Ausbau von grundständigen Studiengängen einen Aufschwung erlebt (Lademann et al. 2016). Der Bedarf, akademisch qualifizierte Pflegefachkräfte in der deutschen Pflegepraxis einzusetzen, gilt als unstrittig (u.a. Dieterich et al. 2019; Lüftl und Kerres 2012; Simon und Flaiz 2015; Breuckmann 2014) und wird national wie international mit einer Verbesserung der Pflegequalität in Verbindung gebracht (Aiken et al. 2014; Verband der Pflegedirektorinnen und Pflegedirektoren der Universitätskliniken (VPU) 2015). Die steigenden Anforderungen im Gesundheitswesen, wie Multimorbidität, Chronizität oder eine veränderte Patient_innenrolle, verdeutlichen die Notwendigkeit erweiterter pflegerischer Fähigkeiten und Kompetenzen. Um diesen Anforderungen Rechnung zu tragen, hält der Wissenschaftsrat (2012) die hochschulische Ausbildung von Pflegekräften für erforderlich. Darüber hinaus wird die „Akademisierung als Strategie der Attraktivitätssteigerung" (Dieterich et al. 2019 S. 4) betitelt, wobei gleichzeitig bisher eher wenige akademisierte Pflegefachkräfte in der Praxis tätig sind (Baumann und Kugler 2019).

Mit Inkrafttreten des Pflegeberufegesetzes (PflBG) im Jahr 2020 wurde die hochschulische Pflegeausbildung (§ 37), neben einer klassischen Berufsausbildung, erstmalig als reguläres berufszulassendes und ausbildungsintegrierendes Hochschulstudium gesetzlich verankert. Unterschiedlich strukturierte duale Modellstudiengänge (Lademann et al. 2016), zumeist in Kooperation mit Pflegeschulen, wurden durch die gesetzlichen Regelungen mit dem Ziel einer Vereinheitlichung und Qualitätssicherung der hochschulischen Qualifikation abgelöst.

Um den erwünschten Mehrwert hochschulischer Absolvent_innen für das Gesundheitssystem zu erzielen, ist es essenziell, dass diese in der Praxis Stellen mit geeigneten Aufgabenprofilen erhalten und ausfüllen. Nur dadurch können die akademischen Kompetenzen in der Pflegepra- xis Anwendung finden (Lüftl und Kerres 2012; DarmannFinck 2016).

Die Einmündung von akademisierten Pflegefachkräften in das Arbeitsfeld stellt ein ambivalentes Unterfangen dar (Lüftl und Kerres 2012). Pflegedienstleitungen befürchten, keinen Mehrwert aus der Unterstützung der Studierenden $\mathrm{zu}$ erhalten, wenn diese nach dem Bachelor-Abschluss ein weiteres Studium aufnehmen und dem Arbeitsmarkt nicht zur Verfügung stehen. Baumann und Kugler (2019) bestätigen durch ihre Untersuchung diese Annahme. Mehr als die Hälfte der befragten Bachelor-Absolvent_innen nahmen ein weiteres Studium auf oder streben dieses an. Simon und Flaiz (2015) begründen diesen Sachverhalt damit, dass in den Praxisfeldern Unklarheiten darüber bestehen, welche Handlungsfelder und Kompetenzprofile durch das Qualifikationsniveau von grundständig akademisierten Pflegefachkräften in der Praxis abgedeckt werden. Außerdem stehen subjektive Überzeugungen, wonach sich der Bachelor-Abschluss und die Pflegetätigkeit am Patient_innenbett widersprechen, der Einmündung von akademisierten Pflegekräften entgegen (Lüftl und Kerres 2012). Weiter bestehen Unklarheiten und Unsicherheiten darüber, in welchen Handlungsfeldern akademisierte Pflegefachkräfte in Zukunft eingesetzt werden, welche Verantwortlichkeiten und Handlungsspielräume sie innehaben und wie sie entlohnt werden (Darmann-Finck 2016; Büker und Strupeit 2016; Lehmeyer und Schleinschok 2011; Fesenfeld 2015; Simon und Flaiz 2015; VPU 2015; Hundt und van Hövell 2015). Für die Professionalisierung der Pflege ist es jedoch elementar, dass die Akademisierung des Pflegeberufes auch in der Pflegepraxis ,ankommt“ (Baumann und Kugler 2019 S. 14). Die vom Wissenschaftsrat (2012) angestrebte Akademisierungsquote in der Pflege ist noch lange nicht erreicht. Aktuellere Zahlen liegen hier z.B. aus Niedersachsen vor. In diesem Bundesland weisen lediglich 3\% der registrierten Pflegefachkräfte einen akademischen Abschluss vor (Pflegekammer Niedersachsen 2018). Vor diesem Hintergrund erscheint die Debatte um Handlungsfelder, -spielräume und eine entsprechende Pro- 
filbildung von Aufgaben akademisierter Pflegefachkräfte umso relevanter.

Laut der Verbleibstudie der Absolventinnen und Absolventen der Modellstudiengänge in Nordrhein-Westfalen (VAMOS), die sich zum Ziel gesetzt hat, den „beruflichen Verbleib von Absolvent_innen nach dem Abschluss eines Modellstudiums in den betreffenden Gesundheitsfachberufen umfassend zu beschreiben" (Dieterich et al. 2019 S. XII), gelingt die Berufseinmündung bei dem Großteil der Befragten problemlos (Dieterich et al. 2019). In die Untersuchung einbezogen wurden neben der Berufsgruppe der Gesundheits- und Krankenpflege auch therapeutische Berufsgruppen sowie die Hebammenkunde (Dieterich et al. 2019). Zu den zentralen Erkenntnissen der Erhebung zählt, dass mehr als die Hälfte der befragten Pflegekräfte nach Studienabschluss im Setting Krankenhaus verortet werden können und überwiegend klientennah tätig sind (Dieterich et al. 2019). In die Studie einbezogen wurden neben der Absolvent_innenperspektive auch die der Arbeitgeber_innen. Letztere sind zu $98 \%$ zufrieden mit der Entscheidung, hochschulische Absolvent_innen einzustellen (Dieterich et al. 2019).

Grundsätzlich zeigen Absolvent_innen pflegebezogener Studiengänge oftmals eine geringe Orientierung in Bezug auf mögliche Handlungsfelder und Aufgabenbereiche nach dem Bachelor-Abschluss (Simon und Flaiz 2015), so auch die Pflege-dual-Studierenden der FH Münster. Dieterich et al. (2019 S. 7) weisen darauf hin, dass „Daten zum Verbleib der Absolvent_innen im Beruf und ihre[r] berufliche[n] Zufriedenheit fehlen“. In den letzten Jahren wurden zunehmend Ergebnisse von Absolvent_innenbefragungen (z.B. Zieher und Ayan 2016), Verbleibstudien (z.B. Büker und Strupeit 2016; Dieterich et al. 2019) und Berufseinstiegsstudien (z.B. Mogg 2017; Baumann und Kugler 2019) mit Bezug zur akademischen Pflege(aus)bildung veröffentlicht. Die Absolvent_innen des Studiengangs Pflege dual der FH Münster wurden bei bisherigen Erhebungen aufgrund der additiven Studienstruktur nicht berücksichtigt.

Bundesweit existieren unterschiedliche klinisch-pflegerische Studiengangmodelle (Lademann et al. 2016). Der duale Studiengang B. Sc. Pflege an der FH Münster, auf den sich die vorliegende Studie bezieht, wird nicht als Modellstudiengang deklariert und entsprechend nicht in VAMOS (Dieterich et al. 2019) einbezogen. Dies begründet eine gesonderte Erhebung zum Verbleib, dessen Erkenntnisse im Weiteren dargestellt und diskutiert werden.

\section{Zielsetzung und Fragestellung}

Vor dem Hintergrund fehlender Erkenntnisse in Bezug auf die Studierenden der FH Münster sowie skizzierter offener Fragenstellung zielte die vorliegende Untersuchung darauf ab, Hinweise zum Berufsverbleib und zur Arbeitszufriedenheit der Absolvent_innen der FH Münster zu generieren. Dementsprechend wurde eine onlinegestützte Befragung zum Verbleib der Absolvent_innen des Studiengangs Pflege dual B. Sc. der FH Münster konzipiert und durchgeführt. Die leitenden Forschungsfragen der Studie lauteten: In welchen Handlungsfeldern und Arbeitsbereichen arbeiten die Absolvent_innen nach dem Bachelor-Abschluss Pflege dual an der FH Münster und wie zufrieden sind sie damit? Darüber hinaus war die akademische Weiterqualifizierung von Interesse. Das zentrale Ziel der Verbleibstudie lag darin, Erkenntnisse über die Berufsperspektiven der Studierenden hinsichtlich (a) der Handlungsfelder und Arbeitsbereiche, (b) der subjektiven Zufriedenheit und (c) der (akademischen) Weiterqualifizierung zu gewinnen.

\section{Methodisches Vorgehen}

Die Konzeption des Fragebogens (Kirchhoff et al. 2010) erfolgte literaturbasiert, erfahrungsbezogen und in Anlehnung an die VAMOS-Befragung (Dieterich et al. 2019). Der Onlinefragebogen, erstellt mit EvaSys (evasys GmbH, Lüneburg, Deutschland), wurde nach interner Konsentierung mit 4 pflegewissenschaftlich wie methodisch versierten Kolleg_innen modifiziert und angewendet. Die Befragung fand 2019 als Querschnittserhebung statt und soll zukünftig im Sinne einer Trendstudie wiederholt werden (Bortz und Döring 2016).

Der Fragebogen besteht aus insgesamt 38 Fragen, die in 7 Fragenblöcke (Tab. 1) unterteilt sind. Es handelt sich größtenteils um geschlossene (Multiple- und SingleChoice-)Fragen (Kirchhoff et al. 2010), ergänzt um Freitextfelder und einzelne offene Fragen. Bei Fragen zur Zufriedenheit wurde eine 4-stufige Rating-Skala verwendet. Im Kontext der Befragung wurde unter der Kategorie Abschluss auch Feedback zur Befragung selbst eingeholt. Der Zeitumfang der anonymisierten Befragung wurde mit ca. $10 \mathrm{~min}$ veranschlagt.

Hervorzuheben ist, dass die Befragten durch eine Filterführung je nach aktueller beruflicher Situation nicht alle Items sämtlicher Kategorien beantworten mussten. Gab eine_r der Befragten beispielsweise an, kein weiteres Studium aufgenommen zu haben, so wurden die Fragen der Kategorie Teil B: Studium nicht einbezogen.

Zielgruppe der Befragung waren die Absolvent_innen des Studiengangs B. Sc. Pflege dual an der FH Münster der Abschlussjahrgänge 2016-2018. Damit wurden alle Absolvent_innen seit Initiierung des Studienganges einbezogen, um eine Vollerhebung zu erreichen. Die initiale Kontaktaufnahme erfolgte per E-Mail durch die Autorinnen. Das Anschreiben beinhaltete Angaben zu Aufbau und Ziel der Befragung sowie Kontaktmöglichkeiten für Rückfragen. Die 
Tab. 1 Fragenblöcke mit Anzahl der Fragen

\begin{tabular}{ll}
\hline Fragenblock/Kategorie & $\begin{array}{l}\text { Anzahl der } \\
\text { Fragen }\end{array}$ \\
\hline $\begin{array}{l}\text { Soziodemografische Merkmale } \\
\text { Angaben zum Studium }\end{array}$ & 2 \\
$\begin{array}{l}\text { Teil A: Berufliche Tätigkeit } \\
\text { Stellensuche und Stellenantritt nach Studien- } \\
\text { abschluss }\end{array}$ & 3 \\
$\begin{array}{l}\text { Aktuelle Beschäftigungssituation } \\
\text { Teil B: Studium }\end{array}$ & 5 \\
$\begin{array}{l}\text { Weiterführendes Studium } \\
\text { Teil C: Berufliche Zukunftsperspektive }\end{array}$ & 12 \\
$\begin{array}{l}\text { Zufriedenheit und Ziele } \\
\text { Abschluss }\end{array}$ & 10 \\
Gesamt & 5 \\
\hline
\end{tabular}

Kriterien der informierten Zustimmung nach Schnell und Dunger (2018) wurden im Anschreiben berücksichtigt. Aufgrund der freiwilligen Teilnahme, der Anonymisierung der Daten sowie der Möglichkeit des Abbruches der Onlinebefragung waren keine ethischen Bedenken erkennbar.

Die Befragung wurde in einem Zeitraum von 3 Wochen im Februar 2019 durchgeführt, in der Mitte des Befragungszeitraumes erfolgte eine Erinnerung. Insgesamt wurden 52 Alumni angeschrieben; die Rücklaufquote betrug $57,7 \%$ (30 Befragungen).

Die gewonnenen Daten wurden deskriptiv mittels SPSS 25 ausgewertet (Ostermann und Wolf-Ostermann 2010) und Freitextantworten entsprechend der Häufigkeit ihrer Nennungen gebündelt und kategorisiert.

\section{Ergebnisse}

Für die Ergebnisdarstellung sind 30 Fragebogen erfasst worden. Aufgrund der Filterführung und fehlender Werte wurden nicht alle Fragen von allen Absolvent_innen beant- wortet. Um ein möglichst differenziertes Antwortspektrum zu erhalten, wurden auch die Daten mit fehlenden Angaben zu einzelnen Items berücksichtigt. Nachfolgend werden zentrale Ergebnisse der Befragung dargestellt.

Ein Großteil der Befragten war weiblich $(82,8 \%$ von $n=29)^{1}$. Den größten Anteil der beruflichen Ausbildung nahm die Gesundheits- und Krankenpflege mit $80 \%$ ein. Über $90 \%$ der Befragten waren zum Erhebungszeitpunkt beruflich tätig, mitunter begleitend zu einem weiterführenden Studium (Tab. 2). Die Teilnehmenden waren zwischen 20 und 34 Jahre alt; der Mittelwert betrug 26,4 Jahre. Die meisten Befragten gehören dem Abschlussjahrgang 2017 an $(50 \%$ von $n=30)$.

\section{Teil A: berufliche Tätigkeit (Handlungsfelder und Arbeitsbereiche)}

Die Ergebnisse zur beruflichen Tätigkeit gliedern sich in die Bereiche Berufseinstieg (Stellensuche und Stellenantritt nach Studienabschluss) sowie Arbeitssetting und Tätigkeitsfelder (Aktuelle Beschäftigungssituation).

\section{Stellensuche und Stellenantritt nach Studienabschluss}

In Bezug auf die berufliche Tätigkeit und die Wahl des Arbeitgebers zeigte sich, dass die größte Gruppe diejenigen Absolvent_innen darstellten, die nach dem Studienabschluss ein neues Tätigkeitsfeld belegten. Knapp ein Drittel der Befragten (32,1\% von $n=30$ ) wechselte dazu den $\mathrm{Ar}$ beitgeber und 35,7\% blieben bei ihrem vorherigen Arbeitgeber. Lediglich 14,3\% hatten nach dem Studienabschluss das gleiche Tätigkeitsfeld beim gleichen Arbeitgeber inne, 17,9\% bei einem neuen Arbeitgeber. Der Bewerbungsprozess und der Übergang ins Berufsleben verliefen bei den Befragten sehr unterschiedlich. Der Großteil $(28,6 \%$ von $n=28$ ) erlebte einen problemlosen Bewerbungsprozess

Tab. 2 Charakteristika der Befragten

\begin{tabular}{llll}
\hline Charakteristika der Befragten & Anzahl $(n)$ & Anteil in Prozent \\
\hline Geschlecht & Weiblich & 29 & 82,8 \\
& Männlich & 30 & 17,2 \\
Berufliche Ausbildung & Gesundheits- und Krankenpflege & & 80 \\
& Gesundheits- und Kinderkrankenpflege & 30 & 16,7 \\
Derzeitige & Altenpflege & & 3,3 \\
Beschäftigung & Beruflich tätig & & 56,7 \\
& In einem Studium & 3,3 & 40 \\
\hline
\end{tabular}

$\overline{1}$ Die Angabe $n=\mathrm{x}$ bezieht sich jeweils auf die Gesamtanzahl der Antworten für ein Item, aus der sich die jeweiligen Prozentangaben ergeben. 
Abb. 1 Berufliche Funktion/ Aufgabengebiet (Mehrfachnennung möglich)

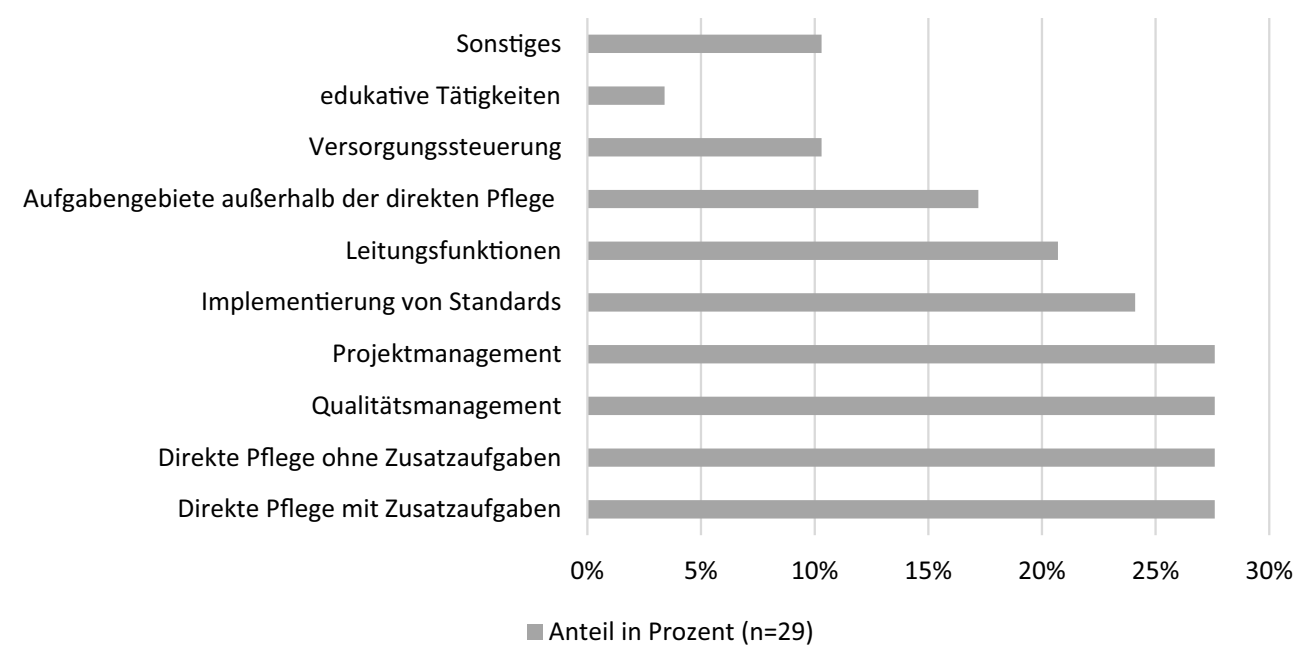

täts- und Projektmanagement sowie die Arbeit in der direkten Pflege mit und ohne Zusatzaufgaben mit jeweils 27,6\% (von $n=29$ ).

Nach subjektiver Einschätzung der Hälfte der Befragten überwogen klientennahe Tätigkeiten in der direkten pflegerischen Versorgung (50\% von $n=26$ ) andere Aufgaben. Konkret arbeiteten 19,2\% ausschließlich im Rahmen von klientennahen Tätigkeiten, bei 23,1\% überwogen andere Tätigkeiten, und lediglich 7,7\% übten im Rahmen ihrer beruflichen Arbeit gar keine klientennahen Tätigkeiten aus. $\mathrm{Zu}$ den Tätigkeiten ohne Patient_innenkontakt zählten u.a. das Schreiben eines Qualitätsmanagementhandbuches, Projektplanungen oder Mitarbeiterschulungen. Bei der Mehrzahl bestand das aktuell ausgeübte berufliche Aufgabenfeld bereits bei Eintritt in die Berufstätigkeit. Nur 18,5\% gaben an, dass ihr Aufgabengebiet in der Einrichtung neu erschlossen wurde. Die Frage nach der Zufriedenheit mit ihrer beruflichen Tätigkeit konnten die Befragten auf einer Skala von 1 (in hohem Maße zufrieden) bis 6 (gar nicht zufrieden) beantworten. Der Mittelwert der Antworten lag bei $2,7$ (SD $\pm 1,4$; von $n=28)$. Die meisten Absolvent_innen waren demnach eher zufrieden, jedoch gaben $7,1 \%$ an, gar nicht zufrieden zu sein.

\section{Teil B: Studium}

Von den Befragten, die sich für ein weiterführendes Studium entschieden haben, nahmen 23,1\% (von $n=13$ ) ein weiteres Bachelor-Studium auf, insbesondere in den Bereichen Pflegepädagogik und Pflegemanagement. Über $80 \%$ hatten sich zum Zeitpunkt der Befragung für ein Masterstudium entschieden. Die gewählten bzw. anvisierten Studiengänge sind äußerst heterogen. Sie reichten von Pflegemanagement, -pädagogik und -wissenschaft über Gerontologie bis hin zu Public Health. Für die Aufnahme des Studiums war bei über der Hälfte der Befragten $(53,8 \%$ von $n=13)$ eine Nachqualifizierung notwendig, bei knapp 38,5\% wurde 
der duale Bachelor-Abschluss vollständig anerkannt. Die Absolvent_innen, die ein weiterführendes Studium aufgenommen haben, belegten dieses überwiegend in Vollzeit $(61,5 \%$ von $n=13), 23,1 \%$ studierten berufsbegleitend und jeweils $7,7 \%$ in Teilzeit oder einer sonstigen Organisationsform. Entsprechend dieser Heterogenität variierten auch die Gründe für die Aufnahme eines weiteren Studiums. Sie reichten von der Fundierung der beruflichen Ausrichtung über den Wunsch nach mehr Hintergrundwissen bis hin zu der Absicht, eine Tätigkeit im Pflege- oder Gesundheitsmanagement oder der Pflegepädagogik aufzunehmen. Hinzu kamen Einstellungsvoraussetzungen im Ausland, Hoffnung auf zusätzliche berufliche Chancen und Perspektiven, die Möglichkeit zu promovieren oder beruflich forschen zu können. Auch die Wünsche nach weiteren Entwicklungsmöglichkeiten oder einem gesundheitlich bedingten Ausstieg aus der direkten Pflege zählten zu den Gründen für die Aufnahme eines weiteren Studiums (jeweils eine Nennung).

\section{Teil C: berufliche Zukunftsperspektiven}

Mehr als die Hälfte der Befragten gaben an, konkret darüber nachzudenken, die berufliche Situation zu verändern (55,2\% von $n=29)$. Dies wurde u. a. mit fehlenden Möglichkeiten der Integration von Studieninhalten in die Pflegepraxis begründet $(21,4 \%$ von $n=14)$. Auch der Wunsch, dem belastenden Pflegealltag zu entkommen, wurde als Begründung angeführt $(57,1 \%)$. Bei den Absolvent_innen, die ein weiteres Studium nach dem Bachelor aufnahmen, diente die derzeitige berufliche Tätigkeit häufig der finanziellen Sicherung.

In Bezug auf weitere Ziele der beruflichen Karriere zeigte sich an erster Stelle der Wunsch nach einem weiterführenden Studium $(64,3 \%$ von $n=28)$, gefolgt von der Zielsetzung, zukünftig in einer Leitungsfunktion $(53,6 \%)$ oder in der Beratung (64,3\%) tätig zu sein. Darüber hinaus wurde eine Lehrtätigkeit in Aus-/Fort- und Weiterbildung von $42,9 \%$ der Befragten als attraktiv angesehen. Nicht wenige Befragte strebten eine Promotion $(21,4 \%)$, die Arbeit als klinische_r Expert_in (17,9\%) oder im Qualitätsmanagement $(39,3 \%)$ an. Zudem wurden als zukünftige Handlungsfelder Praxisanleitung (17,9\%), Forschung und Entwicklung $(28,6 \%)$ sowie die Berufspolitik $(32,1 \%)$ in Betracht gezogen.

Von den befragten Absolvent_innen blickten 13 zum Zeitpunkt der Befragung bereits auf einige berufliche Erfolge zurück, beispielweise wurden verschiedene Fortbildungen sowie ein Traineeprogramm genannt. Außerdem lernten Absolvent_innen unterschiedliche/neue Bereiche im Gesundheitswesen kennen oder absolvierten Auslandspraktika. Als berufliche Erfolge beschrieben sie zudem im Freitext die Übernahme der Projektleitung für das Entlassma- nagement, eine Tätigkeit als studentische Hilfskraft in der betrieblichen Gesundheitsförderung, den Einstieg in den Intensivpflegebereich, die Erstellung eines Qualitätsmanagement-Handbuches, die Aufnahme einer beruflichen Tätigkeit im Ausland, das Pflegetraining für Angehörige oder aber die Verantwortung für die Dokumentation, eine Weiterbildung im Bereich Palliative Care sowie im Management von spezifischen Versorgungsfällen (jeweils eine Nennung).

\section{Abschluss}

Im freien Abschlussstatement berichteten 2 der Absolvent_innen (von $n=4$ ), dass das absolvierte duale Bachelor-Studium sie insgesamt auf einem sehr guten Niveau auf ein nachfolgendes, wissenschaftliches Studium vorbereitet hat - auch im Vergleich mit anderen Student_innen. Eine befragte Person äußerte, die Aufnahme spezieller klinischpflegerischer Positionen und die Etablierung spezifischer Rollen sei stark vom persönlichen Engagement und der individuellen Überzeugungsfähigkeit abhängig.

\section{Diskussion und Ausblick}

Hinsichtlich der Forschungsfrage, in welchem Arbeitssetting sich die Absolvent_innen nach dem BachelorAbschluss Pflege dual wiederfinden, zeigt sich ein eindeutiges Bild. Die meisten Pflegenden sind nach ihrem Bachelor-Abschluss in einem Akutkrankenhaus tätig. Dieses Ergebnis entspricht den Erkenntnissen von Zieher und Ayan (2016). Die Arbeitsfelder ambulanter Pflegedienst, stationäre Pflegeeinrichtung, Reha- sowie Beratungsreinrichtungen werden kaum oder nicht genannt. Um gerade auch diese pflegerischen Bereiche auf die komplexen aktuellen und zukünftigen Bedarfe und Bedürfnisse der Klient_innen, Angehörigen und Pflegenden selbst vorzubereiten, besteht hier dringender Handlungsbedarf aufseiten von Politik und Leistungserbringern. Diese Tendenz entspricht Fleischmanns (2013) Annahme, dass die Forderung nach akademisierten Pflegekräften mit Zunahme der Spezialisierung der Leistungserbringung steigt. Eine Akademisierung klinisch Pflegender, die sich überwiegend auf die stationäre Akutpflege bezieht, geht sowohl an den Entwicklungen der gesundheitlichen Versorgungslandschaft als auch an den individuellen Bedarfen und Bedürfnissen zu pflegender Menschen und ihrer Familien vorbei. Um dem entgegenzuwirken, sollten Studiengänge selbstkritisch ihre Ausrichtung dahingehend prüfen und ggf. anpassen. Am Fachbereich Gesundheit der FH Münster findet derzeit eine entsprechende Überarbeitung des Studienprogrammes statt. Zudem gilt es, in Zusammenarbeit mit den genannten Sektoren Konzepte zu entwickeln, die die Integration 
akademisierter Pflegekräfte fördert (z.B. Robert Bosch Stiftung 2019).

Die meisten der Befragten arbeiten in der direkten Pflege, zu je gleichen Anteilen mit oder ohne Zusatzaufgaben. In Bezug auf die direkte Pflege lassen sich hier konkrete Übereinstimmungen mit den von Darmann-Finck (2016) formulierten Aufgabenprofilen hochschulisch ausgebildeter Pflegender erkennen, welche sich auf die direkte Versorgung, die Fallsteuerung, Pflegeprozessverantwortung, Qualitätsentwicklung und Anleiten und Beraten von Patient_innen sowie Kolleg_innen beziehen (Darmann-Finck 2016). Inwieweit sich die tatsächlich durchgeführte Arbeit von der einer Pflegefachkraft mit beruflicher Pflegeausbildung unterscheidet, ist an dieser Stelle nicht zu erkennen. Vielmehr bedarf es hierzu weiterer Forschung, beispielswiese um spezifische Rollen- und Aufgabenprofile akademisierter Pflegekräfte zu konzipieren bzw. weiterzuentwickeln. Auch VAMOS betont die Wichtigkeit, Handlungsspielräume durch geeignete Stellenprofile auszuschöpfen und die Rahmenbedingungen anzupassen, damit die erweiterten Kompetenzprofile der Absolvent_innen zur Geltung kommen können (Dieterich et al. 2019). Dem Ausstieg aus der Pflege aufgrund von ,fehlenden Stellen für Pflegeakademiker und [dem] Wunsch nach einem ,Mehrwert' des Studiums" (Zieher und Ayan 2016, S. 48) könnte dadurch entgegengewirkt werden. Der Umstand, dass viele Befragte selbst nach Abschluss des Studiums über Unklarheiten bezüglich einer beruflichen Verortung berichten sowie der Sachverhalt, dass viele Absolvent_innen sich ihre Stellen selber „schaffen“ müssen, um ihre akademischen Kompetenzen einbringen zu können, unterstreicht entsprechenden Handlungsbedarf.

Grundsätzlich zeigten sich die Befragten recht zufrieden mit ihrer Entscheidung für das Bachelor-Studium und ihrer bisherigen beruflichen Laufbahn. Im Zusammenhang mit den Angaben zum gewählten Masterstudium und den Antworten auf die Frage nach gewünschten inhaltlichen Schwerpunkten des absolvierten Pflege-dual-Studiums lässt sich ein starker Wunsch nach managementorientierten Inhalten und Vertiefungen ablesen, was den Ergebnissen von Zieher und Ayan (2016) entspricht. Vor dem Hintergrund, dass der Studiengang Pflege dual als klinisch orientierter Bachelor-Studiengang konzipiert wurde und sich dadurch von den in der Pflege historisch lange Zeit vorherrschenden Studienrichtungen der Pflegepädagogik und des Pflegemanagements (Lademann et al. 2016) unterscheidet, ist davon auszugehen, dass dieses Bedürfnis auf falsche Erwartungen an das Studium oder eine unüberlegte Studiengangwahl zurückzuführen ist. Fehlende Rollenvorbilder sowie die bisher nahezu gänzlich fehlenden klinischen Pflegestudiengänge auf Masterniveau befördern vermutlich ebenfalls eine Orientierung in Richtung Pflegemanagement, -pädagogik oder in weitere bereits etablierte Bereiche. Letzteres kann auch den Trend zur Aufnahme eines weiteren Bachelor-Studiums erklären, denn möglicherweise konnten die Befragten dadurch notwendige Voraussetzungen für einen Masterstudiengang erlangen. Nicht immer ist eine Durchlässigkeit in pflegebezogene Masterstudiengänge gewährleistet.

Der Wunsch nach akademischer Weiterqualifizierung ist in diesem Kontext kohärent und der Ausstieg aus der klientennahen Pflegepraxis konsekutiv (Zieher und Ayan 2016). Dies steht den Bestrebungen zur Einrichtung pflegebezogener Studiengänge im Kern entgegen. Eine aktive Bearbeitung dieser Dynamik scheint sowohl vonseiten der Hochschule, der Ausbildungseinrichtungen als auch der Praxiseinrichtungen in sämtlichen Pflegesettings angezeigt.

Der Wunsch nach akademischer Weiterqualifizierung zeigt sich in besonderem Maße in der (beabsichtigten) Aufnahme eines weiterführenden (Master-)Studiums. Auch die VAMOS-Ergebnisse bescheinigen vielen Absolvent_innen ein konkretes Interesse an einem weiteren Studium. Von besonderem Interesse sind dabei klinisch ausgerichtete Masterstudiengänge (Dieterich et al. 2019) oder Managementstudiengänge (Zieher und Ayan 2016). Stehen den Absolvent_innen keine entsprechenden Angebote zur Verfügung, besteht die Gefahr des Ausstieges aus der beruflichen Pflege, was den von Lüftl und Kerres (2012) skizzierten Befürchtungen aufseiten der Pflegedienstleitungen Rechnung tragen würde, dass die Investition in Bachelor-Studierende nicht lohnenswert erscheint, wenn diese im Anschluss dem Arbeitsmarkt nicht zur Verfügung stehen. Hier gilt es grundsätzlich, die Kopplung zwischen Studium und Verwertungsinteresse der Pflegepraxis kritisch zu diskutieren.

Das primäre Ziel klinisch orientierter Pflegestudiengänge, die Absolvent_innen mit erweiterten Handlungskompetenzen auszustatten und so angemessen auf die Versorgung von Klient_innen vorzubereiten, konnte laut den VAMOSErgebnissen erreicht werden (Dieterich et al. 2019). Die Ergebnisse der vorliegenden Befragung zeigen ein ähnliches Bild. Gleichzeitig sind vor dem Hintergrund der Bestrebungen hin zu einer höheren akademischen Qualifizierung auf Masterniveau Überlegungen hinsichtlich eines geeigneten „skill“ und ,grade mix“ in den unterschiedlichen Pflegesettings geboten.

Knapp 30\% der Befragten geben an, nach ihrem Bachelor-Abschluss einen reibungslosen Bewerbungsprozess erlebt und eine Stelle entsprechend ihrer Qualifizierung gefunden zu haben. Daher stellt sich die Frage, inwieweit es für einen gelungenen Berufseinstieg erforderlich ist, den Qualifikationsprofilen entsprechende Tätigkeitsprofile zu schaffen, wie Baumann und Kugler (2019) es fordern. Keiner der Befragten gibt an, Vorbehalte gegenüber der akademischen Qualifizierung erlebt zu haben. Dies lässt hoffen, dass durch aktuelle und zukünftige Entwicklungen im Personalmanagement der Anteil der reibungslosen Übergänge 
in die Pflegepraxis ansteigt. Auf diese Absolvent_innen ausgerichtete Personalentwicklungskonzepte sollten flächendeckend implementiert werden, um das Potenzial der Akademisierung für die Pflege nutzbar zu machen (Baumann und Kugler 2019).

\section{Limitation}

Die Konzeption der Verbleibstudie und ihre Ergebnisse werden durch den Umstand limitiert, dass sowohl die Entwicklung der Befragung als auch ihre Auswertung parallel zur Berufstätigkeit der Autor_innen in ihrer jeweiligen Funktion im Kontext des fokussierten Studiengangs erfolgten. Methodische Schwachstellen sind daher vornehmlich auf fehlende Ressourcen zurückzuführen. Hervorzuheben sind die Nutzung eines literatur- und erfahrungsbasiert konstruierten Fragenbogens ohne Pretest sowie eine ausschließlich deskriptive Datenauswertung. Die dargestellten Ergebnisse zu Handlungsfeldern und Arbeitsbereichen der Absolvent_innen sind aufgrund der geringen Fallzahl nicht repräsentativ, jedoch lassen die Ergebnisse Tendenzen erkennen und erlauben erste Schlussfolgerungen zum Berufsverbleib und zur Zufriedenheit.

\section{Schlussfolgerungen}

Resümierend verdeutlicht die Studie, dass ein erfolgreicher Abschluss des Bachelor-Studiums Pflege dual an der FH Münster den Absolvent_innen vielfältige berufliche Entwicklungstendenzen ermöglicht. Der hohen Motivation zur Weiterentwicklung und -qualifizierung der Absolvent_innen gilt es, zukünftig gezielt zu begegnen. Um das Potenzial der Absolvent_innen gewinnbringend zu nutzen und ihnen Handlungsspielräume zu eröffnen, bedarf es weiterhin innovativer (Personal-)Entwicklungskonzepte.

Funding Open Access funding enabled and organized by Projekt DEAL.

Open Access Dieser Artikel wird unter der Creative Commons Namensnennung 4.0 International Lizenz veröffentlicht, welche die Nutzung, Vervielfältigung, Bearbeitung, Verbreitung und Wiedergabe in jeglichem Medium und Format erlaubt, sofern Sie den/die ursprünglichen Autor(en) und die Quelle ordnungsgemäß nennen, einen Link zur Creative Commons Lizenz beifügen und angeben, ob Änderungen vorgenommen wurden.

Die in diesem Artikel enthaltenen Bilder und sonstiges Drittmaterial unterliegen ebenfalls der genannten Creative Commons Lizenz, sofern sich aus der Abbildungslegende nichts anderes ergibt. Sofern das betreffende Material nicht unter der genannten Creative Commons Lizenz steht und die betreffende Handlung nicht nach gesetzlichen Vorschriften erlaubt ist, ist für die oben aufgeführten Weiterverwendungen des Materials die Einwilligung des jeweiligen Rechteinhabers einzuholen.

Weitere Details zur Lizenz entnehmen Sie bitte der Lizenzinformation auf http://creativecommons.org/licenses/by/4.0/deed.de.

\section{Literatur}

Aiken LH, Sloane DM, Bruyneel L, Van den Heede K, Griffiths P, Busse R et al (2014) Nurse staffing and education and hospital mortality in nine European countries: a retrospective observational study. Lancet 383:1824-1830. https://doi.org/10.1016/S01406736(13)62631-8

Baumann AL, Kugler C (2019) Berufsperspektiven von Absolventinnen und Absolventen rundständig qualifizierender Pflegestudiengänge: Ergebnisse einer bundesweiten Verbleibstudie. Pflege 32:7-16

Breuckmann M (2014) Qualifikation für alle - Bildung im Gesundheitswesen. In: Kaufhold M, Rosowski E, Schürmann M (Hrsg) Bildung im Gesundheitsbereich. Forschung und Entwicklung zur beruflichen und hochschulischen Bildung. LIT, Münster, S 85-96

Büker C, Strupeit S (2016) Pflege-dual-Absolventen: Potenzial wird genutzt. Schwest Pfleg 55:92-95

Darmann-Finck I (2016) Aufgabenfelder hochschulisch ausgebildeter Pflegender. Pflegezeitschrift 69:362-364

Döring N, Bortz J (2016) Forschungsmethoden und Evaluation in den Sozial-und Humanwissenschaften, 5. Aufl. Springer, Berlin https://doi.org/10.1007/978-3-642-41089-5

Fesenfeld A (2015) Alle müssen ihre Hausaufgaben machen. Schwest Pfleg 54:21-23

Fleischmann N (2013) Arbeitsmarktperspektiven für Absolventen pflegebezogener Studiengänge. In: Zängl P (Hrsg) Pflegeforschung trifft Pflegepraxis. Springer, Wiesbaden, S 91-109 https://doi.org/ 10.1007/978-3-658-02573-1_6

Dieterich S, Hoßfeld R, Latteck ÄD, Bonato M, Fuchs-Rechlin K, Helmbold A, große Schlarmann J, Heim S (Hrsg) (2019) Verbleibstudie der Absolventinnen und Absolventen der Modellstudiengänge in Nordrhein-Westfalen (VAMOS) - Abschlussbericht. Bochum 2019. https://www.mags.nrw/sites/default/files/asset/ document/vamos_abschlussbericht_hsg_endversion_publikation. pdf. Zugegriffen: 30. Dez. 2020

Hundt N, van Hövell C (2015) Akademisierung in der Pflege: Aktueller Stand und Zukunftsperspektiven. Disserta, Hamburg

Kirchhoff S, Kuhnt S, Lipp P, Schlawin S (2010) Der Fragebogen: Datenbasis, Konstruktion und Auswertung. VS, Wiesbaden

Lademann J, Latteck Ä-D, Mertin M, Müller K, Müller-Fröhlich C, Ostermann R, Thiehorn U, Weber P (2016) Primärqualifizierende Pflegestudiengänge in Deutschland: Eine Übersicht über Studienstrukturen, -ziele und -inhalte. Pflege Ges 21:330-345

Lehmeyer S, Schleinschok M (2011) Primärqualifizierende Pflegestudiengänge: Eine systematische Analyse dualer und grundständiger Pflegestudienmodelle in Deutschland. Jacobs, Lage

Lüftl K, Kerres A (2012) Ich denk mal, dass andere Bereiche [...] die mit Handkuss aufnehmen. Pflegewissenschaft 14:39-50

Mogg C (2017) Berufseinstiegsstudie von akademisch ausgebildeten Gesundheits- und Krankenpflegepersonen: Absolventenbefragung des Bachelor-Studienganges Gesundheits- und Krankenpflege. Pädagog Gesundheitsber 4:18-23

Ostermann R, Wolf-Ostermann K (2010) Statistik in Sozialer Arbeit und Pflege. Oldenbourg, München https://doi.org/10.1524/ 9783486599473

Pflegekammer Niedersachsen KDÖR (2018) Bericht zur Lage der Pflegefachberufe in Niedersachsen. Erste Datenauswertung aus dem Pflegefachberuferegister der Pflegekammer Niedersachsen. https://www.pflegekammer-nds.de/files/downloads/onlineBericht.zur.Lage.der.Pflegefachpersonen.in.Nds.2018.pdf. Zugegriffen: 30. Dez. 2020

Robert Bosch Stiftung (2019) $360^{\circ}$ Pflege. Qualifikationsmix für den Patienten. https://www.qualifikationsmix-pflege.de/. Zugegriffen: 30. Dez. 2020

Schnell MW, Dunger C (2018) Forschungsethik: Informieren - reflektieren - anwenden. Hogrefe, Bern 
Simon A, Flaiz B (2015) Der Bedarf hochschulisch qualifizierter Pflegekräfte aus Sicht der Praxis: Ergebnisse einer Expertenbefragung. Pflege Ges 20:154-172

VPU Verband der Pflegedirektorinnen und Pflegedirektoren der Universitätskliniken (2015) Einsatz akademisch ausgebildeter Pflegefachpersonen in der Praxis. http://www.vpu-online.de/de/pdf/ presse/2015-05-29_abschlussbericht.pdf. Zugegriffen: 30. Dez. 2020

Wissenschaftsrat (2012) Empfehlungen zu hochschulischen Qualifikationen für das Gesundheitswesen, Berlin. Drs. 2411-12. http:// www.wissenschaftsrat.de/download/archiv/2411-12.pdf. Zugegriffen: 30. Dez. 2020

Zieher J, Ayan T (2016) Karrierewege von Pflegeakademikern: Ergebnisse einer bundesweiten Absolvent*innenbefragung zu Ausbildung, Studium und Beruf. Pflege Ges 21:47-63

Hinweis des Verlags Der Verlag bleibt in Hinblick auf geografische Zuordnungen und Gebietsbezeichnungen in veröffentlichten Karten und Institutsadressen neutral. 